\title{
Percepción de los docentes sobre el abandono escolar y su práctica en el aula en bachilleratos tecnológicos de Baja California
}

\author{
How teachers perceive school dropout and their own \\ practice in the classroom in technological highschools in \\ Baja California
}

DOI: https://doi.org/10.32870/dse.v0i20.576

\section{Karla María Díaz López.* Cecilia \\ Osuna Lever**}

\begin{abstract}
Resumen
En este estudio se describe la opinión de 91 docentes con respecto al abandono escolar en bachilleratos tecnológicos en Baja California, México. Asimismo, se identifican algunos aspectos de su práctica. Se aplicó un cuestionario de opción múltiple para identificar la opinión de los profesores respecto al abandono escolar y medir algunos indicadores de su quehacer en el aula. Los datos se analizaron mediante estadística descriptiva. El ausentismo escolar, la reprobación y las dificultades de aprendizaje son las principales condicionantes que los participantes asocian al abandono; en contraste, las actividades que menos realizan los profesores son: el seguimiento a estudiantes cuando obtienen bajas calificaciones, preocuparse por ellos cuando faltan a clase y conocer detalles de sus vidas. Este hallazgo es significativo pues los propios docentes atribuyen que el ausentismo y las dificultades de aprendizaje son las causas del fenómeno, aunque ellos no dan total atención y seguimiento a dichos aspectos.
\end{abstract}

Palabras clave: abandono escolar - reprobación - dificultades de aprendizaje - bachilleratos.

\begin{abstract}
This study describes the opinions of 91 teachers regarding drop-out in technological high schools in Baja California, Mexico, and identifies some aspects of their practice. A multiple choice questionnaire was applied to identify the opinion of teachers regarding school dropout and to measure some indicators of their work in the classroom. The data was analyzed through descriptive statistics. School absenteeism, failure and learning difficulties are the main factors that participants associate with dropout. In contrast,
\end{abstract}

\footnotetext{
* Doctora en Ciencias Educativas. CETYS Universidad. Líneas de investigación: Abandono escolar, evaluación educativa, diseño de instrumentos y práctica docente. karla.diaz@cetys.mx

** Doctora en Ciencias Educativas. CETYS Universidad. Líneas de investigación: Abandono escolar, educación y valores, ética docente, enseñanza de las matemáticas. cecilia.osuna@cetys.mx
} 
the activities that teachers do least are follow-up to students when they get low grades, being concerned about them when they miss class, and learning about their lives. This finding is significant because the teachers themselves attribute that phenomenon to absenteeism and learning difficulties, although they do not give their full attention and follow-up to these aspects.

Keywords: school dropout - class failure - learning difficulties - high school.

\section{Introducción}

El abandono escolar ${ }^{1}$ representa un fenómeno socioeducativo de gran complejidad, ya que da cuenta de la eficacia de los sistemas educativos y, a su vez, del funcionamiento de la sociedad y el desarrollo de la economía. Según Tarabini, Curran, Montes y Parcerisa (2018), el abandono escolar repercute en la desigualdad de oportunidades y, desde luego, en el derecho a la Educación; en Estados Unidos y en el ámbito latinoamericano se presenta con mayor frecuencia entre los 16 y 24 años de edad, mientras que en la Unión Europea se ubica en un rango muy similar, entre los 18 y 24 años (Hauser y Anderson, 2011).

Durante la última década, los países miembros de la Organización para la Cooperación y el Desarrollo Económico (OCDE) han progresado de manera considerable en la reducción de la tasa de abandono escolar en bachillerato, es decir, la cantidad de jóvenes que no culmina la Educación Media Superior (EMS) ha disminuido. A pesar de los esfuerzos emprendidos, en promedio, aproximadamente uno de cada seis jóvenes entre 25 y 34 años no ha terminado este nivel educativo (OCDE, 2016).

Esta problemática conlleva diversas consecuencias ya que, por una parte, los jóvenes enfrentan dificultades para encontrar un trabajo estable; de acuerdo con González (2016), afecta negativamente su formación educativa, personal y social. A su vez, repercute no solo en el sistema educativo, sino que compromete a la sociedad en su conjunto, ya que genera rezago, exclusión y desigualdad; además, comprende un detonante importante del fenómeno nini (Tuirán y Ávila, 2012). Así pues, queda claro que el abandono escolar se configura como una de las problemáticas socioeducativas que demandan mayor atención en México.

Desde la década de los noventa, Gómez-Villanueva (1990) identificó que el abandono escolar presenta diversas manifestaciones previas, tales como ausentismo escolar, bajo rendimiento académico, acreditaciones extemporáneas, reprobación, repetición y rezago escolar, a lo cual denominamos circuito problemático. En la presente investigación se concibe el abandono escolar como el resultado de un proceso progresivo de ruptura y desencuentro del estudiante con la escuela (Mena, Fernández-Enguita y Riviére, 2010).

1 Fenómeno también denominado deserción escolar en el ámbito mexicano.

2 Termino alusivo a los jóvenes que ni estudian, ni trabajan.

Diólo pos 
Comúnmente, este constructo se explica en función de una serie de factores o variables que suelen agruparse en dos amplios ámbitos: 1. Individual y familiar, en el que se identifican principalmente la motivación e implicación académica, el coeficiente intelectual, edad, adicciones, violencia, embarazo, sexo, involucramiento de los padres, capital cultural, nivel socioeconómico de la familia (Székely, 2015; Weiss, 2015). En particular, algunos autores relacionan el abandono con las clases sociales media y alta (Pérez, 2010), al apoyo otorgado por la familia (Cazorla, Franco, Torres y Archundia, 2016), a la pobreza (Martínez-González y Álvarez-Blanco, 2005), a la falta de vocación (Barbería, 2009). Y 2. Institucional y escolar; en este ámbito destacan variables tales como: programas educativos obsoletos y acceso limitado y desigual a la educación (Tuirán y Ávila, 2012), la organización de la estructura educativa de las escuelas, lineamientos curriculares y métodos de enseñanza, (Rodríguez y Leyva, 2007). Por su parte, el Instituto Nacional de Evaluación Educativa (INEE, 2017) reconoció la influencia de la oferta educativa, la desigualdad en la calidad de los servicios educativos y los mecanismos de acceso, la modalidad y el turno; asimismo, se relaciona con el bajo rendimiento académico debido a prácticas pedagógicas ineficientes, la formación docente limitada y condiciones laborales precarias, infraestructura y equipamiento insuficiente, incompatibilidad entre la cultura juvenil y escolar. Al respecto, Bowers, Sprott y Taff (2013), así como Tukudane, Minnaert, Zeelen y Kanyandago (2015), concluyeron que el elevado número de estudiantes por profesor, la falta de experiencia o una actitud poco adecuada hacia los estudiantes, son factores que inciden directamente en el abandono escolar. De forma semejante, hacia otros investigadores apuntan aspectos relacionados con el papel que desempeña el contexto escolar, en el que se hace especial énfasis en la actuación docente en cuanto a su relación con los estudiantes (D’Alessandre, 2017; Solís, Rodríguez y Brunet, 2013).

En México, se han realizado estudios de alcance nacional (Instituto Nacional de Estadística y Geografía - INEGI, 2009; Instituto Nacional para la Evaluación de la Educación - INEE, 2015; Secretaría de Seguridad Pública - SSP, 2012) y también investigaciones particulares entre las que destacan Cazorla et al. (2016), Farfán, Morales y Johansuly (2016), González (2016), Linares, Gárate, López y González (2012), Román (2013) y Rubio (2015). Si bien en estos estudios se ha reportado la influencia de diversos factores o variables asociadas con el fenómeno en cuestión - destacando los individuales y sociofamiliares-, los informes resultan muy generales y parece soslayarse el papel que desempeña el centro escolar (Tarabini, Curran, Montes y Parcerisa, 2015) y, en particular, se considera de manera limitada el quehacer de los docentes respecto al abandono escolar (Tarabini, 2015), así como su rol ante la problemática en cuestión.

En la literatura iberoamericana se cuenta con pocos estudios sobre el tema. No obstante, en particular destaca lo realizado por Villafaña y Beltrán (2017), en el contexto educativo mexicano; estos autores reportaron que los profesores otorgan un peso importante al ámbito familiar y socioeconómico de los estudiantes, por encima del contexto escolar. Por su parte, Marcano y Ro- 
dríguez (2015), en Venezuela, reportaron que los docentes están deshabituados a la aplicación de técnicas para identificar rasgos de los posibles estudiantes desertores en educación superior.

A decir de Estrada, Alejo y Cervera (2018), los docentes cumplen una función muy importante en la promoción del interés y el arraigo de los estudiantes a la escuela, pues pueden cuidar el ambiente para el aprendizaje. Está comprobado que cuando en el clima del aula impera el aislamiento, la competencia, fragmentación y tensión, los estudiantes se ven perjudicados. Se esperaría que los profesores se conecten con la vida de los estudiantes, los escuchen y compartan con ellos. Así, queda evidenciada la relevancia del papel docente y su influencia en el aprendizaje, lo cual puede atenuar la incidencia del abandono escolar.

\section{El desafío del abandono escolar en Educación Media Superior}

En el Programa Sectorial de Educación 2013-2018 se reconoce la necesidad de la apertura de nuevas escuelas y mejora de las existentes, y se asume como acción prioritaria reducir al mínimo el número de estudiantes que abandonan sus estudios en este nivel educativo. Para ello se plantean algunas cuestiones específicas tales como: desarrollar vínculos con el sector productivo, garantizar la calidad de los planes y programas de estudio, lograr una comunicación estrecha con las familias para que apoyen el proceso formativo de sus hijos, y en particular, se considera fundamental atender el desarrollo profesional de los docentes, con lo que se apuesta a reducir los factores de riesgo que influyen sobre el abandono (SEP, 2013). Aunado a estos pronunciamientos, en 2012 se estableció por decreto la obligatoriedad de este nivel educativo y se fijó como plazo para su cumplimento el ciclo escolar 2021-2022.

En el ámbito latinoamericano y de acuerdo con Reirmers (2002), el carácter obligatorio de la EMS genera un efecto contrario al esperado, ya que los jóvenes de los sectores más desprotegidos que antes no tenían acceso a dicho nivel educativo ingresan, pero suelen abandonar sus estudios, lo que se asocia directamente a las desventajas socioeconómicas. En el caso de México, el bachillerato es el nivel educativo en el que se reportan los más altos índices de abandono escolar (12.1\%). Relacionado con ello, se registra una reprobación de $13.7 \%$, una eficiencia terminal de $66.6 \%$ y la cobertura de $76.6 \%$ (SEP, 2018).

Con base en lo enunciado por Tuirán y Hernández (2016), la disminución de la tasa de abandono escolar en este nivel educativo ha sido muy lenta, lo cual da cuenta de las dificultades para encarar este problema. En 2000 la tasa era de casi 19\%, y apenas cayó a 15\% en 2012. La disminución de dicha problemática en este nivel educativo es una tarea que se asume como prioritaria, tanto porque registran tasas altas - muy superiores a las de primaria y secundariacomo por el lamentable truncamiento de la trayectoria educativa de miles de jóvenes. Asimismo, el abandono comprende una de las vías más agudas a las que se anclan el fracaso escolar y la exclusión educativa.

Diólopos año 11 | número 20 | enero-junio 2020| ISSN 2007-2171 
Cabe referir que en 2013 se inició con la capacitación a directores de los planteles públicos a fin de identificar las mejores prácticas para combatir este problema, incluido el desarrollo de un sistema de alerta temprana, que consiste en dar seguimiento continuo y sistemático del ausentismo y el desempeño académico de los estudiantes (SEP, 2017). Los jóvenes que están en esta situación son candidatos a recibir una "beca contra el abandono" de la Secretaría de Educación Pública y con frecuencia se les apoya en el programa de tutorías, estrategia dirigida a atender el rezago, la deserción y los bajos índices de eficiencia terminal, permitiendo la conformación del perfil del estudiante.

Así pues, parece reconocerse que el abandono no es un hecho inevitable, que sus causas no solo son externas a la escuela y que, en consecuencia, las comunidades educativas deben implementar acciones a fin de garantizar la permanencia de los jóvenes. Para lograrlo, se requiere superar, entre otras cuestiones, los aprendizajes poco significativos, la desactualización curricular y la gestión didáctica rutinaria, factores que provocan escaso interés, aburrimiento y desmotivación de los estudiantes (Tuirán y Hernández, 2016).

De acuerdo con Miranda e Islas (2016), hoy en día se cuenta con un marco normativo robusto, que sostiene y promueve el derecho a una Educación Media Superior de calidad y con equidad. Por ello, las acciones de política pública que se desarrollen en México son fundamentales para garantizar el derecho, el acceso y la permanencia en la EMS.

\section{La profesionalización de la práctica docente en la Educación Media Superior: el caso de México}

En el contexto mexicano, la Subsecretaría de Educación Media Superior se encarga de generar acuerdos que fueron plasmados en la Reforma Integral de la EMS vigente desde 2008. En el Acuerdo 447, publicado en el Diario Oficial de la Federación, se estipulan las competencias docentes; a través de estas se pretende cumplir con el objetivo 13 del eje 3, asentado en el Plan Nacional de Desarrollo 2013-2018. Dicho eje dicta que se debe "Fortalecer el acceso y la permanencia en el sistema de enseñanza media superior, brindando una educación de calidad orientada al desarrollo de competencias", en particular, en la estrategia 13.1 se señala que "se asegurará que los profesores tengan las competencias didácticas, pedagógicas y de contenido de sus asignaturas que requieren para un desempeño pedagógico adecuado" (Gobierno de la República, 2012).

En consonancia, en el Programa Sectorial de Educación 2013-2018, en su Objetivo 1:"Elevar la calidad de la educación para que los estudiantes mejoren su nivel de logro educativo, cuenten con medios para tener acceso a un mayor bienestar y contribuyan al desarrollo nacional", en los numerales 1.8 y 1.10 se comprometen como estrategias "definir un perfil deseable del docente", así como "instaurar mecanismos y lineamientos sistemáticos con base en criterios claros para la certificación de competencias que contribuyan a conformar una planta académica de calidad". 
En 2017, el INEE dio a conocer el documento Cinco directrices para mejorar la permanencia escolar en la educación media superior (INEE, 2017); la tercera de estas consiste en el fortalecimiento de las competencias docentes para generar interacciones pedagógicas pertinentes a las necesidades educativas de los jóvenes.

Así pues, los docentes, además de centrarse en sus acciones pedagógicas orientadas a facilitar la adquisición de aprendizajes de las asignaturas que imparten, también deben apoyar de manera integral la formación de los jóvenes. Un buen docente debe tener altas expectativas sobre el desempeño de sus estudiantes y, partiendo del punto en el que los recibe, llevarlos a que desarrollen su máximo potencial. De conformidad con la Reforma Educativa (2012), los concursos para el ingreso al Servicio Profesional Docente deben asegurar la idoneidad de los profesores. La SEP, a través del Nuevo Modelo Educativo (SEP, 2017), dio a conocer el marco para el desarrollo de la formación docente continua, el cual expresa que, para poder dar un servicio en el sistema, debe de:

1. Cumplir los fines de la educación.

2. Llevar a los alumnos a alcanzar el perfil de egreso de la educación obligatoria.

3. Asumir el enfoque humanista del planteamiento curricular.

4. Desarrollar y planear la enseñanza conforme a la noción de aprendizajes clave.

5. Asumir que el desarrollo personal y social de los estudiantes, y en especial, el desarrollo de sus habilidades socioemocionales, es tan importante como la formación académica.

6. Aprovechar la autonomía curricular para adecuar plenamente la enseñanza a los intereses y las necesidades específicas del alumnado.

En el estudio realizado por Fernández, Mena y Riviére (2010) se menciona que una de las causas de abandono más importantes es la formación del profesorado. En este sentido, Lorenzo, Muñoz y Beas (2015) concluyeron que la grave carencia formativa presente en la formación inicial docente figura como factor causante del abandono escolar. Estos hallazgos coinciden con lo reportado por Santana-Hernández, Marchena-Gómez Martín-Quintana y Alemán-Falcón (2018). Mientras que, Tarabini (2015) expuso que los docentes ubican como causantes del abandono la falta de interés de la familia y la asociación de los problemas de aprendizaje con aspectos biológicos. A su vez, en la investigación realizada por González-Rodríguez, Vieira y Vidal (2019) se evidenció que los factores que los docentes más relacionan con este fenómeno son aquellos de carácter individual y familiar.

Sin duda, resulta pertinente considerar la percepción de los docentes respecto a las variables que inciden en el abandono escolar, ya que a su vez son espectadores y protagonistas de este complejo fenómeno (González-Rodríguez, et al., 2019). 
El panorama descrito hasta ahora hace patente la importancia que tienen los docentes ya que a ellos les compete la loable tarea de facilitar el logro de aprendizajes significativos, generar interés y motivación en los procesos académicos, y a su vez, son los actores educativos que tienen mayor contacto y cercanía con los estudiantes. Por ello, tanto su competencia profesional como la consideración de sus opiniones ante la compleja problemática del abandono escolar, resultan de suma relevancia. Bajo estas consideraciones, en la presente investigación se plantean los siguientes objetivos:

- Identificar la percepción de una muestra de docentes respecto a los factores que influyen en el abandono escolar en los Colegios de Estudios Científicos y Tecnológicos del Estado (CECYTE), ubicados en las tres ciudades más pobladas de Baja California.

- Describir algunos indicadores del quehacer en el aula, de la muestra de docentes que laboran en tres planteles del Colegio de Estudios Científicos y Tecnológicos del Estado (CECYTE) de Baja California.

\section{Método y desarrollo}

Con base en lo antes expuesto, la presente investigación se circunscribe como un estudio descriptivo con una metodología cuantitativa. A continuación, se enuncia el método utilizado.

\section{Participantes}

La población de docentes en los tres planteles para el ciclo escolar 2014 fue de 182, de los cuales participaron 91. La mayoría de los docentes laboran en el plantel de la ciudad de Tijuana, Baja California, México (véase tabla I).

Tabla I. Conformación de la muestra de docentes participantes en cada plantel

\begin{tabular}{|c|c|c|c|c|c|}
\hline \multirow[t]{2}{*}{ Plantel y Municipio } & \multirow{2}{*}{$\begin{array}{c}\text { Población de } \\
\text { docentes }\end{array}$} & \multirow{2}{*}{$\begin{array}{c}\text { Docentes } \\
\text { participantes }\end{array}$} & \multirow[t]{2}{*}{ Porcentaje } & \multicolumn{2}{|c|}{ Sexo } \\
\hline & & & & Femenino & Masculino \\
\hline CECYTE Compuertas (Mexicali) & 40 & 20 & $22 \%$ & 13 & 7 \\
\hline CECYTE E | Florido (Tijuana) & 86 & 51 & $56 \%$ & 16 & 35 \\
\hline CECYTE (Ensenada) & 56 & 20 & $22 \%$ & 13 & 7 \\
\hline Totales & 182 & 91 & $100 \%$ & 42 & 49 \\
\hline
\end{tabular}

Fuente: elaboración propia con base en los resultados de la encuesta.

\section{El cuestionario para docentes}

Está conformado por 23 reactivos agrupados en cuatro dimensiones: I. Datos de identificación, II. Factores asociados al abandono escolar, III. Relación interpersonal con el estudiante, y IV. Práctica pedagógica y desempeño docente. El cuestionario fue construido con base en el modelo 
categorial respecto al abandono escolar, propuesto por Osuna, Díaz-López, Gárate y Contreras (2014), y validado por tres jueces expertos. Dicho modelo se compone de cuatro subcategorías: 1. factores psicológicos, 2. factores personales, 3. factores familiares, y 4. factores del sistema educativo y escolar.

\section{Procedimientos para la recolección y análisis de datos}

Una vez piloteado el instrumento y realizadas las modificaciones necesarias. Los directivos de los tres planteles proporcionaron los correos electrónicos de los docentes, a quienes les fue enviada la invitación para responder el cuestionario en la plataforma electrónica Survey Monkey. Los datos fueron analizados en Statistical Package for the Social Sciences (SPSS) versión 19.

\section{Discusión de resultados}

Enseguida se muestran los resultados producto del análisis de la información generada por medio del cuestionario aplicado. Al respecto, conviene señalar que este no contó con un reporte psicométrico de confiabilidad; no obstante, cuenta con una validación de contenido mediante jueceo.

De la muestra de 91 docentes, $46 \%$ son mujeres y $54 \%$ hombres. En cuanto al turno en que laboran, la mayoría lo hacen por la mañana. Respecto al grado de estudios, profesión y años de experiencia profesional, los resultados se presentan en la tabla II. Destaca que tan solo $25 \%$ cuenta con maestría y 3\% tiene estudios técnicos. La gran mayoría cuenta con una carrera universitaria y solo $10 \%$ son licenciados en Educación.

Tabla II. Máximo grados de estudio, profesión y experiencia docente

\begin{tabular}{|c|c|c|c|c|c|}
\hline Grado de estudios & $\%$ & Profesión & $\%$ & Años de experiencia & $\%$ \\
\hline Licenciatura & $52 \%$ & $\begin{array}{l}\text { Carrera universitaria de cualquier } \\
\text { área de conocimiento }\end{array}$ & $87 \%$ & Entre 6 y 10 años & $47 \%$ \\
\hline Maestría & $25 \%$ & $\begin{array}{l}\text { Licenciado(a) en Educación o } \\
\text { Pedagogía }\end{array}$ & $10 \%$ & Más de 11 años & $45 \%$ \\
\hline Diplomado & $10 \%$ & Maestro(a) normalista & $3 \%$ & Entre 1 y 5 años & $6 \%$ \\
\hline Especialidad & $9 \%$ & & & Menos de 1 año & $2 \%$ \\
\hline $\begin{array}{l}\text { Preparatoria y/o } \\
\text { estudios técnicos }\end{array}$ & $3 \%$ & & & & \\
\hline Doctorado & $1 \%$ & & & & \\
\hline
\end{tabular}

En cuanto a los factores que influyen en el abandono escolar, los docentes consideraron que el ausentismo escolar, la reprobación y las dificultades de aprendizaje son los más importantes (véase tabla III). 
Percepción de los docentes sobre el abandono escolar y su práctica en el aula en bachilleratos tecnológicos de Baja California

Tabla III. Factores que influyen en la deserción estudiantil desde la perspectiva del docente

\begin{tabular}{lll}
\hline & Factores referidos & Porcentaje \\
\hline 1. & Ausentismo escolar & $70 \%$ \\
\hline $\mathbf{2 .}$ & Reprobación continua de materias & $67 \%$ \\
\hline 3. & Dificultades de aprendizaje & $49 \%$ \\
\hline 4. & Falta de apoyo afectivo y educativo de la familia & $39 \%$ \\
\hline 5. & Necesidad de trabajar & $37 \%$ \\
\hline 6. & Influencia negativa de amistades & $35 \%$ \\
\hline
\end{tabular}

Fuente: elaboración propia con base en los resultados.

Cabe referir que los tres primeros factores forman parte del circuito problemático mencionado por Gómez-Villanueva (1990), a su vez, los docentes también identifican la importancia de factores relativos al contexto familiar y social, lo cual tiene consonancia con lo pronunciado por autores como Cazorla et al. (2016) y Román (2013).

Hay que hacer notar que la falta de apoyo afectivo y a su vez educativo, por parte de las familias, se configuró en estos resultados como el cuarto factor para explicar por qué los jóvenes abandonan sus estudios. Según Tarabini (2015), a menudo se culpa a la familia por delegar a la escuela las tareas instructivas, sin considerar las oportunidades reales que esta tiene para llevar a cabo la tarea educativa que el profesorado demanda. Llama la atención que los participantes omiten el papel y responsabilidad que tienen los centros escolares y, desde luego, su propia labor como docentes, lo cual concuerda con lo expuesto por Auwarker y Aruguete (2008), quienes concluyeron que la deserción escolar se percibe a menudo como un elemento fuera del control del profesorado.

En concordancia con estos resultados, Del Burgo (2002) reportó que en España 55.4\% del profesorado asocia el fracaso escolar con poco esfuerzo del alumno, con baja colaboración de las familias (17.1\%), y solamente $11.8 \%$ con el hecho de que los docentes no adaptan las enseñanzas a las necesidades del alumnado.

En el presente estudio, en cuanto al desempeño y práctica docente, la gran mayoría de los docentes (99\%) indicaron que mantienen el orden y el respeto durante las clases; tratan por igual a todos los estudiantes; generan un ambiente propicio para el aprendizaje; dan instrucciones claras para que los estudiantes realicen ejercicios o tareas, y atienden sus dudas. En referencia a las acciones con menor presencia y que se clasifican en el orden de estrategias didácticas para favorecer el aprendizaje, se ubicaron la organización de debates y discusiones entre los estudiantes y la promoción de proyectos diseñados por los alumnos (véase tabla IV). 
Tabla IV. Desempeño y práctica pedagógica de los docentes

\begin{tabular}{l|ccc}
\hline \multicolumn{1}{c|}{ Por lo general en el aula: } & \multicolumn{2}{c}{ Porcentaje } \\
\cline { 2 - 4 } & $\begin{array}{c}\text { Nunca a } \\
\text { casi nunca }\end{array}$ & A veces & $\begin{array}{c}\text { Casisiempre o } \\
\text { siempre }\end{array}$ \\
\hline $\begin{array}{l}\text { Mantengo el orden y el respeto durante las clases } \\
\text { Comunico con claridad las reglas para el desarrollo de las } \\
\text { clases }\end{array}$ & 1 & 0 & 99 \\
Trato por igual a todos los estudiantes & 1 & 1 & 98 \\
\hline Genero un ambiente propicio para el aprendizaje & 1 & 0 & 99 \\
\hline $\begin{array}{l}\text { Doy oportunidad a los estudiantes externen sus ideas } \\
\text { ante los demás }\end{array}$ & 1 & 3 & 96 \\
$\begin{array}{l}\text { Emito instrucciones claras para que los estudiantes } \\
\text { realicen ejercicios o tareas }\end{array}$ & 1 & 0 & 99 \\
Fomento que los estudiantes investiguen ciertos temas & 2 & 4 & 93 \\
\hline $\begin{array}{l}\text { Organizo debates y discusiones para que todos los } \\
\text { estudiantes participen }\end{array}$ & 7 & 21 & 72 \\
\hline $\begin{array}{l}\text { Promueve que los estudiantes diseñen sus propios } \\
\text { proyectos }\end{array}$ & 3 & 10 & 87 \\
\hline $\begin{array}{l}\text { Atiende las dudas de sus estudiantes } \\
\text { Revisa las tareas y les informa sobre sus errores o aciertos }\end{array}$ & 1 & 1 & 99 \\
\hline
\end{tabular}

Nota: Se destaca en negrita los mayores porcentajes

Fuente: elaboración propia con base en los resultados de la encuesta.

En general, se observa que la mayoría de los docentes sí enfocan su quehacer en el aula en los estudiantes $y$, al parecer, mantienen un desempeño que denota sus competencias profesionales. Lo que es favorable, pues en el estudio realizado por Martínez-González y Álvarez-Blanco (2005) la actuación adecuada del profesorado se privilegia como un factor protector del abandono y fracaso escolar.

También se pidió a los docentes que reportaran algunos aspectos que dieran cuenta de la relación interpersonal que mantienen con sus estudiantes; al respecto, destaca que la gran mayoría (arriba de 79\%) los felicitan cuando obtienen buenas calificaciones, los motivan para que continúen estudiando y los alientan para que expresen sus opiniones ante la clase mostrándoles respeto. Este hallazgo revela que al parecer los docentes cuentan con actitudes adecuadas para promover el aprendizaje de sus estudiantes (Solís et al., 2013). Un poco más de la mitad reportó hacer sentir cuidados y apreciados a sus estudiantes. No obstante, solo 64\% "a veces" le da seguimiento a sus aprendizajes cuando obtienen bajas calificaciones, solo $65 \%$ se preocupa cuando los estudiantes faltan, y únicamente $22 \%$ se interesa por conocer detalles de la vida de sus alumnos (véase tabla $\mathrm{V}$ ). 
Tabla V. Relación interpersonal con los estudiantes

\begin{tabular}{|c|c|c|c|}
\hline \multirow{2}{*}{ Como docente: } & \multicolumn{3}{|c|}{ Porcentaje (\%) } \\
\hline & Nunca & A veces & Siempre \\
\hline Doy seguimiento a los alumnos que obtienen bajas calificaciones & 2 & 33 & 64 \\
\hline Pienso que la mayoria son excelentes estudiantes & 8 & 55 & 37 \\
\hline Los felicito cuando obtienen buenas calificaciones & 1 & 9 & 90 \\
\hline Los motivo para que continúen estudiando & 1 & 9 & 90 \\
\hline Suelo preocuparme por saber por qué faltan a clase & 3 & 32 & 65 \\
\hline Cuando tienen algún problema les ofrezco mi apoyo & 2 & 35 & 63 \\
\hline Atiendo sus necesidades haciéndolos sentir cuidados y apreciados & 2 & 41 & 57 \\
\hline $\begin{array}{l}\text { Suelo conocer detalles de sus vidas (familia, intereses personales necesidades } \\
\text { económicas, etc.) }\end{array}$ & 11 & 66 & 23 \\
\hline Los aliento para que expresen sus opiniones ante la clase mostrándoles respeto & 2 & 19 & 79 \\
\hline Comparto con ellos historias de mi vida y los invito a que compartan las suyas & 10 & 40 & 50 \\
\hline
\end{tabular}

Fuente: elaboración propia con base en los resultados de la encuesta.

Tomando en cuenta que los docentes opinan que el ausentismo, la reprobación de materias y las dificultades de aprendizaje comprenden los tres principales factores que se relacionan con la deserción estudiantil, llama la atención que sean precisamente estos aspectos los que suelen desatender o realizar en menor medida.

En el mismo sentido de este hallazgo, Tuirán y Hernández (2017) sostuvieron que será necesario configurar la EMS que México demanda, lo cual implica avanzar hacia el desarrollo e implementación de una nueva escuela, que a su vez responda a los desafíos del siglo XXI; un espacio en el que los jóvenes encuentren sentido a sus aprendizajes y un ámbito donde construyan su identidad, para lo cual los docentes deben asumir la responsabilidad de comprender y acompañar sus procesos, misma que incidiría como factor protector de la deserción.

\section{Conclusiones y prospectiva}

En la misma línea de lo planteado por González-Rodríguez et al. (2019) y a la luz de los resultados obtenidos, la escuela como institución, y en particular los roles desempeñados por los docentes, inciden de manera significativa en el abandono escolar. Destaca que los esfuerzos emprendidos por las autoridades educativas, en coordinación el gobierno del país, para abatir la condición de abandono escolar, parecen no resultar suficientes para garantizar el acceso y, sobre todo, la permanencia y culminación de los estudios en EMS, situación que también señaló Miranda (2018).

En específico, sobre las variables relativas a la institución escolar, se pueden establecer programas de intervención, para lo cual se requiere la colaboración y observación de los docentes, 
así como programas específicos de apoyo dirigidos a estudiantes que lo requieran. Para ello, en primera instancia, es necesario que las escuelas, y en particular los docentes, tomen conciencia de que existen diversas variables vinculadas al abandono.

Respecto al perfil idóneo del docente, los resultados observados en este estudio permitieron detectar que la gran mayoría tiene un perfil profesional diverso (87\%), pero escasa actualización profesional, en particular, solamente $25 \%$ tiene una maestría. Este factor pudiera influir en un limitado manejo de conocimientos de la disciplina de formación, e incluso, aunque el porcentaje no es muy alto (3\%), hay profesores enseñando que no cuentan con una licenciatura. Esto marca una brecha para conocer cuántas y qué tipo de capacitaciones didáctico-pedagógicas han recibido estos docentes, para asegurar una enseñanza eficiente. Hay que identificar lo anterior en congruencia con lo planteado en el Plan Nacional de Desarrollo 2013-2018, que privilegia que los docentes sean competentes en el manejo de estrategias didácticas y de contenido para un desempeño adecuado. Con base en ello, persiste una duda sobre la idoneidad docente (SEP, 2017).

El ausentismo escolar, la reprobación y las dificultades de aprendizaje figuraron como los tres principales factores que desde la perspectiva de los docentes se relacionan con el abandono escolar de estudiantes de EMS. Así pues, se evidencia la presencia del circuito problemático. Estos hallazgos coinciden con la investigación realizada por González, García, Ruíz y Muñoz (2015).

A pesar de que al parecer los docentes están conscientes de ello, en su práctica docente cotidiana otorgan poca atención a los aspectos que se relacionan con estos tres factores, como por ejemplo, identificar el ausentismo escolar o dar seguimiento a los estudiantes con bajas calificaciones. Estos resultados permiten notar que los participantes dejan en segundo término el papel y la responsabilidad que tienen los centros escolares y su propia labor.

En general, se observa que la mayoría de los docentes reportaron que su quehacer en el aula se centra en los estudiantes y en lograr que su desempeño sea adecuado. Sin embargo, para futuros estudios convendría corroborar lo anterior con la percepción de sus estudiantes, e incluso de los directivos, y triangular los datos con base en la observación directa en el aula.

Por otra parte, la escuela debe asumir un rol activo en la gestión de actividades asociadas a la mejora de la práctica docente, tales como: atención a procesos de enseñanza-aprendizaje en el aula, cuestiones relativas a la interacción docente-estudiante, además de vincular la participación de la familia en el centro escolar (comunicación entre padres de familia y docentes). En virtud de lo indicado, sería oportuno que los centros escolares otorguen un acompañamiento al docente, para que realice un adecuado seguimiento a los estudiantes que con cierta regularidad se ausentan de clases, que registran un bajo rendimiento académico y que tienen dificultades para aprender.

Diálo@os sobre Educación año 11 | número 20 | enero-junio 2020| ISSN 2007-2171 
A su vez, y no menos importante, resulta conveniente fomentar la cooperación entre las familias y los centros educativos, ya que las primeras pueden proporcionar a las escuelas información sobre sí mismas que ayudaría a comprender de mejor manera las circunstancias que inciden en el abandono. Asimismo y de acuerdo con Miranda (2018), se requiere pedagogizar el abandono escolar, lo cual implica convertir el problema en un asunto vinculado a la capacidad de los docentes y de la escuela, para generar interacciones pedagógicas significativas con los jóvenes, así como fortalecer las acciones para atender necesidades educativas específicas, con base en las acciones de tutoría y acompañamiento cada vez más profesionales, con capacidad niveladora, pero también de integración académica y psicosocial de los jóvenes. Los hallazgos de esta investigación indican la necesidad de realizar estudios en esta misma línea.

Finalmente, a partir de lo evidenciado en este estudio, se plantea como prospectiva que, si se quiere abatir esta problemática educativa, resultados como estos dan algunas pistas; sin embargo, se debe continuar indagando, añadir estudios que consideren la opinión de los estudiantes y sus familias sobre este fenómeno, con objeto de aportar soluciones integrales reales que tomen en cuenta a todos los sujetos involucrados, para así lograr la disminución efectiva de la deserción escolar.

\section{Referencias}

Auwarker, A.E. y M. Aruguete (2008). Effects of Student Gender and Socioeconomic Status on Teacher Perceptions. Journal of Educational Research, 10, 243-246.

Barbería, J. (2009). Generación 'ni-ni': ni estudia ni trabaja. Periódico El País. http://elpais.com/ diario/2009/06/22/sociedad/1245621601 850215.html

Bowers, A.J., R. Sprott y S.A. Taff (2013). Do We Know Who Will Drop Out? A Review of the Predictors of Dropping out of High School: Precision, Sensitivity, and Specificity. The High School Journal, 96(2), 77-100. doi:10.1353/hsj.2013.0000

Cazorla, L.M., V.J. Franco, L.O. Torres y M.C. Archundia (2016). ¿Quiénes son los estudiantes que abandonan los estudios? Identificación de factores personales y familiares asociados al abandono escolar en estudiantes de educación media superior. Congresos CLABES. http:// revistas.utp.ac.pa/index.php/clabes/article/view/906/933

D’Alessandre, V. (2017). La relación de las y los jóvenes con el sistema educativo ante el nuevo impacto de inclusión en el nivel medio. En N. López, R. Opertti y C. Vargas. Adolescentes y jóvenes en realidades cambiantes. Notas para repensar la educación secundaria en América Latina. Francia: UNESCO.

Del Burgo, M. (2002). El fracaso escolar. Madrid: Acento Editorial. 
Diario Oficial de la Federación (9 de febrero, 2012). Decreto por el que se declara reformado el párrafo primero; el inciso c) de la fracción II y la fracción $\mathrm{V}$ del artículo 3o. y la fracción I del artículo 31 de la Constitución Política de los Estados Unidos Mexicanos.

(29 de octubre, 2008). Acuerdo número 447 por el que se establecen las competencias docentes para quienes impartan educación media superior en la modalidad escolarizada. México: SEP. http://www.sems.gob.mx/work/models/sems/Resource/11435/1/images/5 4 acuerdo 447 comp etencias docentes ems.pdf

Estrada, M., S. Alejo y C. Cervera (2018). El análisis del abandono escolar: la perspectiva del docente y los jóvenes estudiantes. El caso de una escuela del Colegio de Educación Media de la Universidad de Guanajuato. En M. Estrada (coord.). Abandono escolar en la educación media superior en México. Políticas, actores y análisis de casos. México: Universidad de Guanajuato, 181-209.

Farfán, M., M. Morales y V. Johansuly (2016). Factores que influyen en el abandono escolar de estudiantes de preparatoria Sultepec y San Simón de Guerrero.http://ri.uaemex.mx/handle/20.500.11799/58955

Fernández, M., L. Mena y J. Riviére (2010). Fracaso y abandono escolar en España. http://psicopedia.org/wp-content/uploads/2015/04/Fracaso-Escolar.pdf

Gobierno de la República (2012). Plan Nacional de Desarrollo. http://itcampeche.edu.mx/ wp-content/uploads/2016/06/Plan-Nacional-de-Desarrollo-PND-2013-2018-PDF.pdf

Gómez-Villanueva, J. (1990). El rezago escolar en la educación superior: Un breve examen. Perfiles Educativos, 49-50, 14-26, México: UNAM.

González, L.F. (2016). Informe de investigación sobre deserción escolar mediante jerarquización de factores en la UPIIG, del IPN en Silao, Guanajuato. REXE-Revista de Estudios y Experiencias en Educación, 10(19), 33-48. http://www.rexe.cl/ojournal/index.php/rexe/article/viewFile/119/126

González, S., M.P. García, F. Ruíz y J.M. Muñoz (2015). Factores de riesgo del abandono escolar desde la perspectiva del profesorado de Educación Secundaria Obligatoria en Andalucía (España). Profesorado. Revista de Currículum y Formación del Profesorado, 19(3), 226-245.

González-Rodríguez, D., M.J. Vieira y J. Vidal (2019). La percepción del profesorado de Educación Primaria y Educación Secundaria sobre las variables que influyen en el Abandono Escolar Temprano. Revista de Investigación Educativa, 37(1), 181-200. http://dx.doi.org/10.6018/ rie.37.1.343751

Hauser, R. y J. Anderson (2011). High School Dropout, Graduation, and Completion Rates: Better Data, Better Measures, Better Decisions. Washington: The National Academies Press.

INEGI (2009). Encuesta Nacional de Ocupación y Empleo. México: INEGI. http://www.inegi.org.

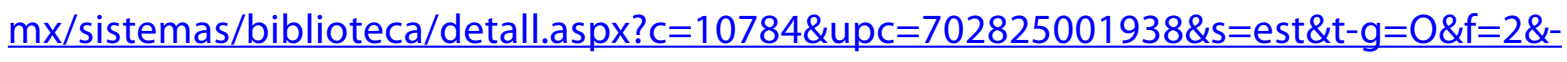
$\mathrm{cl}=\mathrm{O} \& \mathrm{pf}=\mathrm{EnCH} \& \mathrm{ef}=\mathrm{O}$ 
INEE (2017). Directrices para mejorar la permanencia escolar en la educación media superior. México: INEE. http://publicaciones.inee.edu.mx/buscadorPub/P1/F/105/P1F105.pdf

(2015). Panorama educativo en México. Educación básica y media superior. Indicadores del Sistema Educativo Nacional. México: INEE. Recuperado de http://www.inee.edu.mx/images/2015/Panorama-2014/PEM2014-4.pdf

Linares, L., A. Gárate, L. López y C. González (2012). La generación nini: los hijos de la precariedad. Mexicali: Ediciones CETYS.

Lorenzo, J.A., I.M. Muñoz y M. Beas (2015). Modelos de formación inicial del profesorado de Educación Secundaria en España desde una perspectiva Europea. Revista Complutense de Educación, 26 (3), 741-757.

Marcano, Y. y R. Rodríguez (2015). Minería de datos aplicada a la deserción estudiantil. Caso: Licenciatura en Computación-Universidad del Zulia-NPF. Educare, 18(2), 31-51.

Martínez-González, R.A. y L. Álvarez-Blanco (2005). Fracaso y abandono escolar en Educación Secundaria Obligatoria: implicación de la familia y los centros escolares. Aula Abierta, 85.

Mena, L., M. Fernández-Enguita y J. Riviére (2010). Desenganchados de la educación: procesos, experiencias, motivaciones y estrategias del abandono y del fracaso escolar. Revista de Educación, Número extraordinario, 119-145. http://www.revistaeducacion.educacion.es/re2010/re2010.pdf

Miranda, F. (2018). Abandono escolar en educación media superior: conocimiento y aportaciones de política pública. Sinéctica, Revista Electrónica de Educación. doi: 10.31391/S2007-7033(2018)0051$\underline{010}$

y J. Islas (2016). Los jóvenes, sus derechos y la equidad en la educación media superior en México: hacia una agenda de política educativa. Polifonías, Revista de Educación, 5(9), 149-177. http:// www.polifoniasrevista.unlu.edu.ar/sites/www.polifoniasrevista.unlu.edu.ar/files/site/9\%20Miranda\%20e\%20lslas.pdf

OCDE (2016). Panorama de la Sociedad 2016. Un primer plano sobre los jóvenes. La situación de México. OCDE. https://www.oecd.org/mexico/sag2016-mexico.pdf

Osuna, C., K. Díaz-López, A. Gárate y C. Contreras (2014). Estudio de los factores que influyen en el abandono escolar en educación media superior y su relación con la pedagogía de la alteridad. El caso de Baja California, México. En P. Ortega, Educar en la alteridad. España: Editorial REDIPE, 193222. ISBN 9978-958-58278-1-3.

Pérez, I. (2010). Los 'ninis', un nuevo fenómeno social. En Suite101.net. http://www.suite101.net/content/los-ninis-un-nuevo-fenomeno-social-en-mexico-a24692

Reimers, F. (2002). Oportunidades y políticas educacionales en Latinoamérica. En F. Reimers (coord.). Distintas escuelas, diferentes oportunidades. Los retos para la igualdad de oportunidades en Latinoamérica, España: La Muralla.

Rodríguez, L. y P. Leyva (2007). La deserción escolar universitaria, la experiencia de la UNAM. El Cotidiano, 22(142), 98-100. 
Román, M. (2013). Factores asociados al abandono y la deserción escolar en América Latina: Una mirada de conjunto. REICE. Revista Electrónica Iberoamericana sobre Calidad, Eficacia y Cambio en Educación, 11(2), 33-59. http://www.redalyc.org/pdf/551/55127024002.pdf

Rubio, S. (2015). La reprobación, principal factor que origina la deserción escolar en la educación media superior en León, Guanajuato. Reaxion Ciencia y Tecnología Universitaria. http:// reaxion.utleon.edu.mx/Art La reprobacion principal factor que origina la desercion escolar en la educacion media superior en Leon Guanajuato.html

Santana-Hernández, R., R. Marchena-Gómez, J.C. Martín-Quintana y J. Alemán-Falcón (2018). Abandono escolar y formación docente. Aula Abierta, 47(3), 365-372. doi: https://doi. org/10.17811/rifie.47.3.2018.365-372

SSP (2012). Reporte de la Encuesta Nacional de Deserción en la Educación Media Superior. Gobierno de México: SEP. http://www.siguele.sems.gob.mx/encuesta.php

(2013). Programa Sectorial de Educación 2013-2018. México: SEP e Instituto Nacional de Salud Pública (2015). Análisis del movimiento contra el abandono escolar en la educación media superior. http://www.sems.gob.mx/work/models/sems/Resource/11390/6/images/ reporte abandono.pdf

(2017). Formación docente continua. Marco para el desarrollo de la formación docente continua. Educación básica. México: SEP.

(2018). Estadísticas e indicadores educativos por entidad federativa, Sistema Nacional de Información Estadística Educativa. http://www.snie.sep.gob.mx/x entidad federativa.html

Solís, P., E. Rodríguez y N. Brunet (2013). Orígenes sociales, institucionales y decisiones educativas en la trasmisión a la educación media superior. Revista Mexicana de Investigación Educativa, 18(59). http://148.231.10.114:3017/ehost/pdfviewer/pdfviewer?vid=6\&sid=9f1d5ff5cbf5-4efc-8527-67d8421a872f\%40sessionmgr4005\&hid=4109

Székely, M. (2015). Estudio sobre los principales resultados y recomendaciones de la investigación y evaluación educativa en el eje de prevención y atención a la deserción escolar en educación media superior (documento interno). México: INEE.

Tarabini, A. (2015). La meritocracia en la mente del profesorado: un análisis de los discursos docentes en relación al éxito, fracaso y abandono escolar. Revista de la Asociación de Sociología de la Educación, 8(3), 349-360.

M. Curran, A. Montes y L. Parcerisa (2015). El rol de los centros educativos en la prevención del abandono escolar: una aproximación desde la perspectiva micropolítica. Educação, Sociedade \& Culturas (ESC). Special Issue: Education Policies and Early School Leaving.

(2018). Can Educational Engagement Prevent Early School Leaving? Unpacking the School's Effect on Educational Success. Educational Studies, 1-16. DOI: $\underline{10.1080 / 03055698.2018 .1446327}$

Diólo pos 
Tuirán, R. y J. Ávila (2012). Jóvenes que no estudian ni trabajan: ¿Cuántos son? ¿Quiénes son? ¿Qué hacer?. Revista Este País. http://estepais.com/site/?p=37606

y D. Hernández (2016). Desafíos de la educación media superior en México. Revista Este País, 299. http://prepacihuatlan.sems.udg.mx/sites/default/files/adjuntos/01desafios ems mexicorodolfotuiran 0.pdf

Tukudane, C., A. Minnaert, J. Zeelen y P. Kanyandago (2015). A review of enabling factors in support intervention programmes for early school leavers: What are the implications for Sub-Saharan Africa? Children and Youth Services Review, 52, 54-62. doi: 10.1016/j. childyouth.2015.02.0111

Villafaña, L., y A. Beltrán (2017). La deserción en estudiantes de educación superior tres percepción en estudio, alumnos, docentes y padres de familia. Pistas Educativas, 126. http://www. itcelaya.edu.mx/ojs/index.php/pistas/article/viewFile/776/849

Weiss, E. (2015). El abandono escolar en la educación media superior: dimensiones, causas y políticas para abatirlo. En R. Ramírez (ed.). Desafíos de la educación media superior. Ciudad de México: Instituto Belisario Domínguez, 81-160. 